7th International Workshop on Astronomy and

Relativistic Astrophysics (IWARA 2016)

International Journal of Modern Physics: Conference Series

Vol. 45 (2017) 1760046 (8 pages)

(C) The Author(s)

DOI: $10.1142 / S 2010194517600461$

\title{
Quantum Faraday Effect in the Universe
}

\author{
Lídice Cruz Rodríguez \\ Facultad de Física, Universidad de la Habana (FF-UH) \\ San Lázaro y L, Vedado, C.P 10400 La Habana, Cuba \\ lcruz@fisica.uh.cu \\ Aurora Pérez Martínez \\ Departamento de Física Teórica, Instituto de Cibernética, Matemática y Fúsica (ICIMAF) \\ Calle E esq 15 No. 309, Vedado, C.P 10400 La Habana, Cuba \\ Instituto de Ciencias Nucleares, Universidad Nacional Autónoma de México (ICN-UNAM) \\ A. P.70-543, 04510 C. México, México \\ aurora@icimaf.cu \\ Gabriella Piccinelli \\ Centro Tecnológico FES Aragón, Universidad Nacional Autónoma de México \\ Avenida Rancho Seco S/N, Bosques de Aragón, 57130 Nezahualcóyotl \\ Estado de México, México \\ itzmna@unam.mx \\ Elizabeth Rodríguez Querts \\ Departamento de Fúsica Teórica, Instituto de Cibernética, Matemática y Fúsica (ICIMAF) \\ Calle E esq 15 No. 309, Vedado, C.P 10400 La Habana, Cuba \\ Centro de Investigación y de Estudios Avanzados del Instituto Politécnico Nacional \\ (CINVESTAV-IPN) \\ Apdo. Postal 14-740, 07000, C. México, México \\ elizabeth@icimaf.cu
}

Published 15 August 2017

\begin{abstract}
We study the Quantum Faraday rotation starting from the photon self-energy in the presence of a constant magnetic field. The Faraday angle is calculated in the non-degenerate regime and for weak field limit. Two physical scenarios, possibly characterized by these conditions, are the recombination epoch and the jets originated in pulsars. We discuss
\end{abstract}

This is an Open Access article published by World Scientific Publishing Company. It is distributed under the terms of the Creative Commons Attribution 4.0 (CC-BY) License. Further distribution of this work is permitted, provided the original work is properly cited. 


\title{
L. Cruz Rodríguez et al.
}

\begin{abstract}
the resonant behavior that the Faraday angle exhibits in these scenarios and investigate the possibility of detecting cosmic magnetic fields through this resonant mechanism.
\end{abstract}

Keywords: Quantum Faraday Effect.

PACS numbers: 98.35.Eg, 42.25.Bs, 97.60.Gb, 98.80.Es

\section{Introduction}

Faraday effect ${ }^{1}$ is the well known phenomenon where plane polarized light penetrating in a magnetized transparent charged medium and moving parallel to the magnetic field $\mathbf{B}$ rotates its plane of polarization as a consequence of birefringence: the incoming wave splits in two opposite circularly polarized modes moving with different speeds (and frequencies), and the polarization vector rotates.

Faraday Rotation (FR) is a particular case of the general problem of photon propagation in a charged medium ${ }^{2}$. Classical and semiclassical approaches can be applied successfully ${ }^{1}$ in non-relativistic media. However, as was pointed out in Refs. ${ }^{3,4}$, FR effects can be derived from a quantum-relativistic approach which is more appropriate to describe electromagnetic waves under certain extreme conditions that can be present in the Universe.

In Refs. ${ }^{3,4}$ we studied the relation between Faraday angle and Hall conductivity, showing their quantized feature. It was done on the basis of the detailed study of general properties of the photon self-energy and the dispersion equations for photons propagating in the medium, parallel and perpendicular to the magnetic field, considering that the photon self-energy satisfies properties of gauge, Lorentz and CPT invariance ${ }^{2}$. The calculations were done in imaginary time formalism.

The aim of the present paper is to return to the calculations of quantum Faraday rotation in order to further explore the resonance behavior in term of the cyclotron frequency exhibited by the Faraday angle. In particular, we show that in the non-degenerate regime (temperature higher than density) and weak field limit, the resonance is preserved and could be useful to infer the value of the magnetic field. Two relativistic scenarios characterized by these conditions are studied. The first one is a cosmological scenario, where primordial magnetic fields (PMF), possibly present at the recombination epoch, can be estimated in the polarization of the Cosmic Microwave Background (CMB). In this case, we will be able to compare our results with the ones obtained by Planck ${ }^{5}$. The second one is related to an astrophysical context: the jets originated by pulsars. It is interesting to note that, while with other techniques an upper bound is usually estimated for cosmic magnetic fields, with the method we are proposing here the eventual detection of the resonance would allow us to automatically obtain the value of the magnetic field responsible of the phenomenon.

The paper is organized as follows: in Sec. 2 we briefly summarize the photon self-energy and the Faraday angle calculation in presence of a constant and uniform magnetic field. In section 3 we present the angle in the non-degenerate weak field 
limit and discuss the possible application to our cosmological and astrophysical scenarios. Finally, in Sec.4 we state the concluding remarks.

\section{Quantum Faraday Rotation Angle}

The propagation of photons in a relativistic medium at finite temperature and density, in the presence of a constant magnetic field was studied in Ref. ${ }^{2}$. The Maxwell equations in Fourier space are

$$
\left.\left[\left(k_{\perp}^{2}+k_{\|}\right) g_{\mu \nu}-k_{\mu} k_{\nu}+\Pi_{\mu \nu}\left(k \mid A_{\mu}^{e}\right)\right)\right] a_{\nu}(x)=0,
$$

where $k_{\perp}^{2} \equiv k_{1}^{2}+k_{2}^{2}$ and $k_{\|}^{2}=k_{3}^{2}+k_{4}^{2}, A_{\mu}^{e}$ refers to the external magnetic field and $\Pi_{\mu \nu}$ is the photon self-energy which contains all the information related to the interaction with the medium, i.e depends on magnetic field, density and temperature $(\nu, \mu=1,2,3,4)$.

As was discussed in Refs. ${ }^{2,3}$ the polarization tensor in the case of photons propagating parallel to the magnetic field can be expressed in terms of three independent scalars, $I_{t}, I_{r}$ and $I_{s}$

$$
\Pi_{\mu \nu}=\left[\begin{array}{ccc}
I_{t} & i I_{r} & 0 \\
-i I_{r} & I_{t} & 0 \\
0 & 0 & I_{s}
\end{array}\right]
$$

To solve Eq (1) we have to diagonalize (2). The eigenvectors of $\Pi_{\mu \nu}$ satisfy the secular equation $\Pi_{\mu \nu} b_{\nu}^{i}=\kappa_{i} b_{\nu}^{i}$. In the particular case of propagation along the magnetic field there are three non vanishing eigenvalues. Two correspond to transverse modes $b_{\mu}^{\prime}=b_{1} \pm b_{2}$ which describe circularly polarized waves in the plane perpendicular to $B$ with eigenvalues

$$
\kappa_{1,2}=I_{t} \pm I_{r}
$$

There is a third mode $b_{3}$ corresponding to a longitudinal wave which propagates along the magnetic field and $\kappa_{3}=I_{s}$ is the corresponding eigenvalue. The existence of the former two eigenvectors/eigenvalues is the key of the Faraday effect.

The scalars $I_{r}$ and $I_{t}$ in the case of propagation parallel to magnetic field $\left(k_{\perp}=0\right)$ can be written as

$$
\begin{aligned}
I_{r}= & \frac{e^{3} B \omega}{2 \pi^{2}} \sum_{n} \int_{-\infty}^{\infty} d p_{3} \frac{k_{\|}^{2}+2 e B(2 n+1)}{\left(k_{\|}^{2}+2 e B\right)^{2}-4 \omega^{2} \varepsilon_{n}^{2}}-\frac{k_{\|}^{2}+2 e B(2 n-1)}{\left(k_{\|}^{2}-2 e B\right)^{2}-4 \omega^{2} \varepsilon_{n}^{2}} \\
& \times\left(n_{e}\left(\varepsilon_{n}\right)-n_{p}\left(\varepsilon_{n}\right)\right), \\
I_{t}= & -\frac{e^{3} B}{4 \pi^{2}} \sum_{n} \int_{-\infty}^{\infty} \frac{d p_{3}}{\varepsilon_{n}}\left(\left(1-\frac{\left(k_{\|}^{2}+2 e B\right)\left(k_{\|}^{2}+2 e B(2 n+1)\right)}{\left(k_{\|}^{2}+2 e B\right)^{2}-4 \omega^{2} \varepsilon_{n}^{2}}\right)\right. \\
& \left.+\left(1-\frac{\left(k_{\|}^{2}-2 e B\right)\left(k_{\|}^{2}+2 e B(2 n-1)\right)}{\left(k_{\|}^{2}-2 e B\right)^{2}-4 \omega^{2} \varepsilon_{n}^{2}}\right)\right) \times\left(n_{e}\left(\varepsilon_{n}\right)+n_{p}\left(\varepsilon_{n}\right)\right),
\end{aligned}
$$




\section{Cruz Rodríguez et al.}

where

$$
\varepsilon_{n}=\sqrt{p_{3}^{2}+m^{2}+2 e n B}
$$

and $n$ are the Landau numbers, $n_{e}$ and $n_{p}$ are the distribution of electrons and positrons.

The solution of the dispersion relations for the eigenmodes is

$$
k_{ \pm}=\sqrt{\omega^{2}+\kappa_{1,2}}
$$

and the electric field associated to the wave is given by

$$
\mathbf{E}=\left[\frac{i \omega}{\sqrt{2}} A e^{i\left(k_{+} x_{3}-\omega t\right)} \mathbf{e}^{+}+\frac{i \omega}{\sqrt{2}} A e^{i\left(k_{-} x_{3}-\omega t\right)} \mathbf{e}^{-}\right],
$$

where $\mathbf{e}^{ \pm}=\left(\mathbf{e}_{1} \mp i \mathbf{e}_{2}\right) / \sqrt{2}$ are the polarization vectors of the right and left circularly polarized waves, respectively. So, the superposition of both modes leads to an elliptically polarized wave, whose principal axes rotate.

We can define the refraction indices $n_{ \pm}$of the right and left circularly polarized waves, respectively as ${ }^{2}$

$$
n_{ \pm}\left(\omega, k_{3}\right)=\left(1+\frac{\kappa_{1,2}\left(\omega, k_{3}\right)}{\omega^{2}}\right)^{1 / 2} .
$$

The amount of the FR angle, after traveling a distance $L$ in the medium is

$$
\theta_{F}=\frac{1}{2} \omega\left(n_{+}-n_{-}\right) L=\frac{1}{2}\left(\operatorname{Re}\left[k_{-}\right]-\operatorname{Re}\left[k_{+}\right]\right) L,
$$

where we have used the relation $k=n \omega$. Considering expressions (8)-(9) we obtain

$$
\operatorname{Re}\left[k_{ \pm}\right]=\frac{1}{\sqrt{2}}\left[\sqrt{\left(\omega^{2}+\operatorname{Re}\left[\kappa_{1,2}\right]\right)^{2}+\operatorname{Im}\left[\kappa_{1,2}\right]^{2}}+\left(\omega^{2}+\operatorname{Re}\left[\kappa_{1,2}\right]\right)\right]^{1 / 2} .
$$

If

$$
\operatorname{Im}\left[\kappa_{1,2}\right] \ll \omega^{2}+\operatorname{Re}\left[\kappa_{1,2}\right],
$$

and

$$
\operatorname{Re}\left[\kappa_{1,2}\right] \ll \omega^{2},
$$

in the leading order approximation

$$
\operatorname{Re}\left[k_{ \pm}\right] \approx \omega+\frac{\operatorname{Re}\left[\kappa_{1,2}\right]}{2 \omega},
$$

and, according to the relation given in (10), we obtain for the rotated Faraday angle per unit length ${ }^{\mathrm{a}}$

$$
\frac{\theta_{F}}{L} \approx \frac{I_{r}(\hbar \omega+i \Gamma, B)}{2 \hbar(\omega+i \Gamma) c} .
$$

$\Gamma$ is the probability of absorption of the eigenmodes which remains finite at the threshold and depend only on the value of the magnetic field and has been added

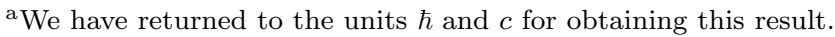


to the total photon energy in the expression obtained for the Faraday angle. It is possible to estimate this correction by using the equation $\Gamma=\frac{I m \kappa_{i}}{2 \operatorname{Re\omega }}$. Then, in order to estimate the amount of Faraday rotation we must evaluate the expression (14).

\section{Faraday Angle for Non-Degenerate and Weak Field Limit}

In the weak field limit $\left(e B \ll m^{2}\right)$ the distance between Landau levels is small $(\sim e B)$. Therefore, we can consider the discrete spectra as a continuum and replace the sum over $n$ in Eq. (4) by an integral. If we consider the non-degenerate limit

$$
n_{e, p} \ll 1, n_{e, p} \rightarrow e^{-(\varepsilon \mp \mu) \beta},
$$

with relativistic spectrum for the particles

$$
\varepsilon=\sqrt{\mathbf{p}^{2}+m^{2}} .
$$

Then

$$
n_{e}-n_{p} \simeq 2 e^{-\varepsilon \beta} \sinh (\mu \beta) .
$$

In the limit of long wave $k_{3} \rightarrow 0$, after some algebra we find for $I_{r}$ the following expression:

$$
I_{r}=A(\mu, \beta)\left(\frac{J}{4}\left(\frac{2 e B+2 \omega^{2}}{3 \omega^{2}}\right) F_{2}(z)-\frac{J^{\prime}}{4}\left(\frac{-2 e B+2 \omega^{2}}{3 \omega^{2}}\right) F_{2}\left(z^{\prime}\right)\right),
$$

where

$$
\begin{aligned}
& J=-\omega^{2}+2 e B ; J^{\prime}=-\omega^{2}-2 e B, \\
& z=\frac{J \beta}{2 \omega} ; z^{\prime}=\frac{J^{\prime} \beta}{2 \omega},
\end{aligned}
$$

and

$$
A=\frac{-2 e^{2} \sinh (\mu \beta)}{\pi^{2} \omega^{2} \beta} .
$$

We have used the following definition

$$
F_{2}(z)=\int_{0}^{\infty} d x \frac{x^{2} e^{-x}}{x^{2}-z^{2}}
$$

and consider two limits:

(1) $\left|z, z^{\prime}\right| \gg 1$, which means

$$
\beta\left(2 e B \mp \omega^{2}\right) \gg 2 \omega .
$$

In this limit, we can approximately consider that the singularity at $x=z$ is out of the integration interval so $F_{2}(z)$ turns into $F_{2}(z) \simeq \frac{2 !}{1-z^{2}}$. Then $\theta_{F}$ takes the form

$$
\frac{\theta_{F}}{L}(\omega, B, \mu, T) \approx \frac{1}{2 c} \frac{16 e^{2}}{3 \pi^{2}} \frac{\sinh (\mu \beta)}{\beta^{3}} \frac{e B+(\omega+i \Gamma)^{2}}{4 e^{2} B^{2}-(\omega+i \Gamma)^{4}} .
$$

Expression (23) shows a resonant behavior at $\omega^{2} \simeq 2 e B$ which could be useful to obtain a value of the magnetic field for fixed frequencies, considering the 


\section{Cruz Rodríguez et al.}

bound (22). In such way the condition to be fulfilled is $\omega>>T$. We analyze below two scenarios, a cosmological one and an astrophysical one, where this approximation makes sense.

(2) $\left|z, z^{\prime}\right|<<1, F_{2}(z) \simeq 1$ and the expression for the Faraday angle becomes

$$
\frac{\theta_{F}}{L}(\omega, B, \mu, T) \approx \frac{8 e^{3} B \mu}{\pi^{2} \omega^{2} \beta},
$$

where we have considered $\sinh (\mu \beta) \sim \mu \beta$. Expression (24) is the well-known semiclassical limit for the Faraday angle ${ }^{6,7}$, proportional to the magnetic field and inverse to the frequency.

\subsection{Primordial magnetic fields and cosmic microwave background radiation}

As is well known, CMB is fundamental for observational cosmology because it is the oldest light in the universe, dating to the epoch of recombination. On the other hand, magnetic fields are present at all scales in the universe, from compact objects ${ }^{8}$, up to clusters of galaxies ${ }^{9}$ and superclusters ${ }^{10}$. The mechanism of generation of these cosmic fields is an open problem and one of the hypothesis is that they could be a remnant of a primordial magnetic field. If this is the case and they were generated at some pre-decoupling epoch, they would have left an imprint on the CMB spectrum, both in temperature and polarization ${ }^{5}$. Among their effects, we are interested in the fact that they induce a Faraday rotation ${ }^{11}$. Different methods are exploited in the Planck report in order to establish constraints on PMF, from which the more stringent are at the level of nanogauss. On the other hand, Faraday rotation of CMB polarization, with Planck power spectra in EE and $\mathrm{BB}$ at $70 \mathrm{GHz}$, give a less restrictive bound: $1.38 \mu G$. We are then interested in exploring the values that can be pinpointed by (23). The temperature of the CMB radiation being $2.73 K$, the condition of $\omega \gg T$ is satisfied for frequencies at least higher than $55 \mathrm{GHz}$, which means fields corresponding to $B>4.5 \times 10^{-6} G$. In particular, for $70 G H z$ we obtain a value of the magnetic field $7.1 \times 10^{-6} G$ which is of the same order than the bound imposed by Faraday rotation from Plank through the semi-classical expression, far from the cyclotron frequency. Unfortunately, the fact that this method obtains values 3 orders higher than the constraint obtained through other mechanisms, renders its application not very suitable for CMB. The point that the cyclotron and the observational frequencies are far apart when studying CMB was already noticed by Ref. ${ }^{12}$. So, we will now turn to another scenario, where both frequencies can be more coincident.

\subsection{Jets from radio-pulsars}

Relativistic pulsar jets is a phenomenon where streams of matter are emitted almost at the speed of light along the axis of rotation of a compact object. The mechanism 
responsible for pulsar jet formation is a matter of debate but it is widely believed that magnetic fields play an important role. The temperature of a typical radiopulsar is $10^{6} \mathrm{~K} \sim 10^{2} \mathrm{eV}$. If the emission is in the X-ray band, as is the case of Crab [13] and Vela [14] pulsars, frequencies are in the range of $3 \times 10^{16}-10^{19} \mathrm{~Hz}$ which admits the condition $\omega \gg T$ imposed by (22). Hence, the Faraday angle (23) for this radiation would be able to detect magnetic field strengths in the range of $10^{6}-10^{9} \mathrm{G}$. These are plausible values for magnetic fields in jets since the strengths on radio pulsar surfaces are of order of $10^{12}-10^{13} G$ (see Ref. ${ }^{15}$ ). We think that it would be important to study the polarization of the radiation received from the jet-pulsar, as is done for AGN-jets ${ }^{16}$, since the detection of the resonance would contribute to build the model for the generation of jets.

\section{Conclusions}

We have studied the quantum Faraday angle for weak field limit and non degenerate gas. The expression has a singularity that allows to estimate precise values of magnetic fields in physical scenarios. In particular, we have considered the possibility of detecting primordial magnetic fields in the CMB and astrophysical magnetic fields present in jets of radio pulsars. In the first case, the frequencies at which the CMB spectrum was observed by the Planck satellite would only allow to detect strengths of primordial fields already excluded by other methods. In the second scenario, observing the polarization of jet radiation in the X-ray band would select magnetic fields in a range of values that could effectively be present in the jet.

\section{Acknowledgments}

L. C. R, A. P. M and E. R. Q. have been partially supported by the grant CB0407 and the ICTP Office of External Activities through NET-35. A.P.M thanks Consejo Nacional de Ciencia y Tecnologia (CONACYT) for the support with the sabbatical Grant 264150 at ICN-UNAM, Mexico, where this work was developed. G. P. B and A. P. M acknowledge the grant PAPIIT-IN117914 from DGAPA-UNAM. ERQ has been also supported by a TWAS-CONACYT 2015 postdoctoral fellowship.

\section{References}

1. M. Faraday, Philos. Trans. R. Soc. London 139, 1 (1846).

2. H. Pérez Rojas and A. E. Shabad, Ann of Phys. 138, 1 (1982); H. Pérez Rojas, Informe Científico Técnico No. 71, junio de 1978; H. Pérez Rojas and A. E. Shabad, Ann. Phys. 121432 (1979); H. Pérez Rojas, J. Exp. Theor. Phys. 76, 1 (1979).

3. L. Cruz Rodríguez, A. Pérez Martínez, H. Pérez Rojas, and E. Rodriguez Querts, Phys. Rev. A 88, no. 5, 052126 (2013). doi:10.1103/PhysRevA.88.052126 [arXiv:1309.6652 [hep-ph]](2013).

4. L. Cruz Rodríguez, A. Péres Martínez, H. Péres Rojas, and E. Rodrigues Querts, Astron. Nachr. 336, 895 (2015). doi:10.1002/asna.201512245 [arXiv:1508.01001 [astroph.HE]](2015). 
5. P. A. R. Ade et al. [Planck Collaboration], A $6 A$ 594, Planck 2015 results XIX (2016), arXiv:1502.01594 [astro-ph.CO](2015).

6. A. K. Ganguly, S. Konar, and P. B. Pal, Phys. Rev. D 60, 105014 (1999). doi:10.1103/PhysRevD.60.105014 [hep-ph/9905206](1999).

7. J. C. D'Olivo, J. F. Nieves, and S. Sahu, Phys. Rev. D 67, 025018 (2003). doi:10.1103/PhysRevD.67.025018 [hep-ph/0208146](2002).

8. P. M. Woods and C. Thompson, Soft Gamma Repeaters and Anomalous X-ray Pulsars: Magnetar Candidates in Compact Stellar X-Ray Sources, eds. W. H. G. Lewin and M. van der Klis (Cambridge Univ. Press, Cambridge, England, 2006) p. 547.

9. T. E. Clarke, P. P. Kronberg, and H. Boehringer, ApJ 547, L111 (2001). DOI:10.1051/0004-6361/201118526, arXiv:1111.6186 [astroph.GA](2011); A. Bonafede, L. Feretti, M. Murgia et al., A 6 A 513, A30 (2010). DOI: 10.1051/0004-6361/200913696, arXiv:1002.0594 [astro-ph.CO](2010).

10. Y. Xu, P. P. Kronberg, S. Habib, and Q. W. Dufton, ApJ 637, 19 (2006). DOI: 10.1086/498336, astro-ph/0509826()2005; E. J. Kim, P. P. Kronberg, G. Giovannini, and T. Venturi, Nature 341, 720 (1989). doi:10.1038/341720a0.

11. A. Loeb and A. Kosowsky, Astrophys. J. 469, 1 (1996). arXiv:astro-ph/9601055(1996).

12. M. Giovannini, Phys. Rev. D 71, 021301 (2005). doi:10.1103/PhysRevD.71.021301. [hep-ph/0410387](2004).

13. Y. Lyubarsky and D. Eichler, Astrophys. J. 562, 494 (2001). doi:10.1086/323436; [astro-ph/0204481](2002).

14. M. Durant, O. Kargaltsev, G. G. Pavlov, J. Kropotina, and K. Levenfish, Astrophys. J. 763, 72 (2013). doi:10.1088/0004-637X/763/2/72; [arXiv:1211.0347 [astroph.HE]](2013).

15. R. C. Duncan, (2003) Magnetars, Soft Gamma Repeaters amd Very Strong Magnetic Fields, http://solomon.as.utexas.edu/magnetar.html (accessed December, 2016); http://solomon. as . utexas . edu/magnetar.html.

16. A. Reichstein and D. Gabuzda, J. Phys. Conf. Ser. 355, 012021 (2012). doi:10.1088/1742-6596/355/1/012021. [arXiv:1109.2507 [astro-ph.CO]](2011). 